\title{
Modeling of Propagation of Road Hazard Information in Sparse Vehicular ad hoc Networks
}

\author{
Sepehr Keykhaie $^{1} \quad$ Saleh Yousefi $^{2} \quad$ Mehdi Dehghan $^{3}$ \\ ${ }^{1}$ Department of Computer and IT Engineering, Chehelsotoon Institute of Higher Education, Isfahan, Iran \\ ${ }^{2}$ Department of Computer, Faculty of Engineering, Urmia University, Orumiyeh, Iran \\ ${ }^{3}$ Department of Computer Engineering and IT, Amir Kabir University of Technology, Tehran, Iran
}

\begin{abstract}
In this paper, we study the propagation of road hazard information to vehicles which enter the hazard segment of a highway in a sparse 1D vehicular ad hoc network (VANET) with store-and-forward mechanism. Store-and-forward is an option for message propagation in sparse vehicular networks where connectivity is intermittent. Upon receiving the message, the vehicle becomes an informed vehicle, it carries the message for a while and then forwards it to the approaching vehicles which are about to enter the highway segment. In this way, a platoon of informed vehicles is formed. We establish an analytical model to obtain the probability that a vehicle receives the message and joins the informed platoon. Moreover, we prove that traffic dynamics increase the reception probability of messages. We find the expected message propagation delay in the platoon using the store-and-forward policy. We also show that the propagation delay in store-and-forward inter-vehicle communications is tightly related to traffic parameters such as traffic flow rate and vehicle speeds on the highway. Results show that for smaller transmission ranges, smaller platoons are formed, the expected message propagation delay in the platoon is low, and it increases very slightly as the traffic flow rate increases. But for larger transmission ranges, larger platoons are formed, the expected delay is high, and it increases remarkably with a small increase in the traffic flow rate. The impacts of some network and traffic parameters such as transmission range, speed of vehicles, and highway speed limits on the message propagation are investigated as well. Finally, the accuracy of the analytical results is evaluated by an extensive simulation study.
\end{abstract}

Keywords: Vehicular ad hoc networks (VANETs), store-and-forward, message propagation delay, informed platoon size, reception probability.

\section{Introduction}

Vehicular ad hoc networks (VANETs) are special types of mobile ad hoc networks (MANETs), where wirelessequipped vehicles communicate with one another and form a network on the road. In VANETs, vehicles can communicate directly without the need of expensive infrastructure. VANETs have some characteristics that distinguish them from the other MANETs, such as having intermittent connectivity due to traffic density variation and restricted mobility pattern, largely one dimensional (1D) or stripe-like topology due to the nature of roadway geometry. Applications of VANET are classified as traffic safety, traffic efficiency and value-added applications ${ }^{[1]}$. Safety applications aim to improve the safety of passengers by exchanging relevant safety messages. Examples are cooperative collision avoidance and other hazard detection warning systems. Traffic efficiency applications aim to optimize the traffic flow (better route selection, better traffic balance, shorter travel time, etc.). Examples for value-added applications include announcement of roadside business (hotels, restaurants, gas stations, etc.) and entertainment applications (collaborative gaming, web surfing, on-line media streaming, etc.).

VANETs encounter two different types of network densities (i.e., density of equipped vehicles) depending on the time of the day and on the market penetration rate (MPR):

1) Dense network is formed by high density of equipped vehicles. In these networks, since connectivity is satisfactory, message propagation is not a challenge and MACrelated problems such as collision and contention are of more concern.

2) Sparse network is formed by low density of equipped vehicles. In these networks, since instantaneous connectivity between vehicles is not always available, message propagation is a big challenge and MAC-related problems are less important due to network sparsity. A store-and-forward scheme is proposed as an option for message propagation in sparse mobile environments. In this paradigm, when a vehicle (node) receives a message, it stores the message in the memory and carries it. Whenever connectivity to the next hop is available, it forwards the message to the new nodes.

Hereinafter, we refer to vehicle trajectory as the moving path from the vehicle's starting position. We refer to stationary traffic state as a traffic state when it does not change over time. In this case, vehicles share the same speed and trajectories are straight and parallel lines and no overtaking 
is allowed in this case. And we refer non-stationary traffic state as a traffic state when trajectory lines intersect one another and overtaking is allowed ${ }^{[2]}$. In unidirectional highway scenarios, which we peruse in this paper, if all vehicles move at the same speed (i.e., stationary traffic), then the inter-vehicle distances remain unchanged. If this distance is greater than the transmission range, then message propagation stops. Therefore, non-stationary traffic is necessary for the message propagation in one-way road scenarios ${ }^{[3]}$.

In this paper, we focus on informing vehicles of a road hazard as they enter the hazard segment on a unidirectional highway with store-and-forward inter-vehicle communications. The nodes enter the hazard segment following a Poisson distribution and move with their chosen speeds. Most of the studies assume a constant number of nodes in a time (space) interval to find the joint distribution of arrival times (distances from the origin). However, in our model the number of nodes is assumed to be random as in reality in our model. Based on this assumption, we find the joint distribution of arrival times, then we obtain the marginal distribution of arrival times which helps us to find the contact probability (i.e., the probability that a vehicle is able to communicate with other vehicles in front of it when it enters the highway segment). We also obtain the probability that a node becomes an informed node as it enters the segment. We also find a simple approximated formula for the contact probability which can be used in finding the average time taken to inform all vehicles in a platoon. Furthermore, we investigate the impacts of some network and traffic parameters such as transmission range, speed of nodes, and road speed limits on the message propagation process.

The remainder of this paper is organized as follows. Section 2 reviews the related works. Section 3 proposed the analytical model. Section 4 presents some numerical and simulation results, followed by conclusions in Section 5 .

\section{Related works}

Recently, the study of connectivity in MANETs has attracted considerable interest. VANETs can be considered as 1D MANETs with all nodes distributed along a straight line, so the results of the studies on 1D mobile networks can be applied to VANETs. Some important performance measures of connectivity in VANETs include: expected connected distance (i.e., cluster length), expected number of vehicles in a connected path (i.e., cluster size), the probability of having at least one communication path between two nodes, the $k$-hop connectivity, and the critical transmission range ${ }^{[4]}$. Some researchers studied the connectivity aspects of VANETs in static networks. The exact connectivity probability of $1 \mathrm{D}$ networks with uniformly distributed nodes was obtained with two different approaches ${ }^{[5,6]}$. But a number of studies exist in relation to network connectivity with mobile nodes. In [7], the distribution of intervehicle distance was found. Based on it, we studied the connectivity metrics such as connectivity distance using the equivalent infinite server queuing model. The impact of speed on connectivity was studied and shown by stochastic ordering techniques. Under the assumption that vehicles' speeds vary according to the wide sense stationary (WSS) ergodic random process, $\mathrm{Wu}^{[8]}$ revealed that the distribution of variable speed nodes asymptotically converges to the distribution of constant speed nodes as $t \rightarrow \infty$, and an exact closed-form connectivity probability expression was obtained. Moreover, some works studied connectivity parameters under more complicated mobility models. Connectivity properties of mobile VANETs were studied in [9] which assumed that vehicles arrive and depart at predefined entry points along a highway. A mobility model using queuing network was proposed in [10] by considering the topology of the road and behavior of vehicles. And at the end, the lower and upper bounds for connectivity probability were presented.

Information propagation in a sparse VANET is typically based on a store-carry-forward scheme ${ }^{[11-14]}$ alike that in a delay-tolerant network ${ }^{[15]}$. More recent works consider this scheme in their model. By assuming a Poisson distribution of nodes along a line of length $L$, the probability of network connectivity on two-way street scenarios using sore-carry-forward routing was investigated in [16]. Based on measured empirical traffic data in [17], the connectivity parameters such as cluster size and cluster length were studied in sparse networks, and the average time taken to deliver a message to the neighboring cluster with the assistance of the opposite traffic was obtained. Kesting et al. ${ }^{[18]}$ investigated message propagation in a store-and-forward scheme called transversal message hopping. Messages are transported by vehicles traveling in the opposite direction when communication link is not available in the original direction and return to the original direction when communication link appears. They found the probability distribution for transmission time and the related message propagation speed between two vehicles driving in a same direction in this mode. In [19], the upper and lower bounds for average information propagation speed were presented for a bi-directional highway scenario. Below the lower bound, which is a function of the traffic density in each direction, data propagated at vehicle speed. In other word, above the upper bound average, message speed quickly increases as a function of traffic density and approaches radio propagation speed. However, as discussed in the previous section, these models are not suitable for one-way street scenarios because they assume all nodes maintain a same constant speed in their models. Considering VANET as a partitioned network with nodes having random speeds, Wu et al. ${ }^{[3]}$ studied the message propagation along a specific road segment of unidirectional highway by informing downstream vehicles in sparse and dense networks. Then, they extended their model for two-way scenarios. However, their model is not appropriate for safety applications where messages (e.g., road hazard messages) are more useful for upstream traffic rather than the downstream traffic. In this paper, we focus on message propagation to upstream vehicles which 
enter the hazardous road segment after the vehicle creating the initial message about the hazard in the segment. On the other hand, for upstream vehicles, the vehicles nearby must receive the message immediately to make decisions in time (e.g., slowing down or changing the lane) as they enter the hazard segment. Other vehicles which are further from the hazard segment also need to receive the message to make decisions based on their distance to the hazard (e.g., detouring). Informing the nearby vehicles which we study in this paper is of more concern, because they have short time to react due to the short distance to the hazard. A distance threshold from the hazard is defined, which depends on the human/vehicle reaction time and travel speed. Vehicles must get informed of the road hazard as they pass the distance threshold and enter the hazard segment. This distance threshold gives drivers a reasonable time to react in time. Here, we aim at informing vehicles of the road hazard, and study of drivers' reactions is out of scope of this paper. However, when a shock wave is generated on the highway (due to leading vehicles' reactions, e.g., slowing down), challenges such as collision between simultaneous transmissions must be addressed in the vehicular communications.

Although there are a number of works on connectivity and message propagation in VANETs, most of them only consider spatial connectivity metrics in their models. In this paper, we also study a temporal metric in our model. The use of vehicles' trajectory lines in a time-space plane simplifies studying spatial-temporal connectivity metrics which is not considered in the aforementioned work.

\section{Analytical model}

\subsection{Definitions and assumptions}

Suppose that $N(t)$ nodes enter a highway segment in the time interval $[0, t]$. MPR is $100 \%$. The first vehicle which enters the highway segment in this time interval is the message source and its arrival time is $S_{1}$. This vehicle is equipped with sensing abilities and generates a message about the road hazard as it enters the hazard segment. Other vehicles, with only communicating abilities, arrive at $S_{i}, i=2,3, \cdots, N(t)$. Also, no two vehicles have the same arrival time, i.e., $S_{1}<S_{2}<\cdots<S_{k}<\cdots$. The vehicles which have received the message are referred to as informed vehicles, other vehicles are uninformed. A sequence of informed vehicles forms an informed platoon. The informed platoon head is referred to the informed vehicle with the largest position coordinate. We also refer to the informed platoon tail as the informed vehicle with the smallest position coordinate form the segment entrance point. The transmission range of each vehicle is $r$. We assume that the vehicle length is negligible in terms of its impact on connectivity. At a light traffic load, corresponding to free-flow state in traffic theory ${ }^{[20]}$, since the network is sparse with a very low connectivity, collision between the simultaneous transmissions is trivial. Therefore, we do not consider the MAC-related problems ${ }^{[7]}$. This assumption helps us to simplify our analysis of message propagation in sparse VANET. Also, we only consider vehicle-to-vehicle communication $(\mathrm{V} 2 \mathrm{~V})$ for the message propagation in our model, which benefits from the low cost and easy deployment. In other word, we do not use base stations for broadcasting messages, albeit we can easily deploy the model to study the message propagation in hybrid architecture, i.e., with the existence of base stations along the roadside.

Since all vehicles share a same speed in a stationary traffic state, when vehicle $k, k \leq N(t)$, enters the highway segment, it receives the message only of the predecessor vehicle (i.e., $k-1$ ) is in its transmission range ${ }^{[17]}$. But, in a nonstationary traffic state, due to random vehicle speeds, positions of the vehicles change, and a vehicle other than vehicle $k-1$ delivers the message to it when vehicle $k$ enters the high way. Consider the example illustrated in Fig. 1, where the message source carries the message, the horizontal line shows the entrance point of the highway, solid lines are vehicle trajectories and positions of the predecessor nodes at a new arrival are depicted with $x$ along the vertical dashed line. Suppose that $k$ is 7 , as node 7 enters the segment, it cannot communicate with its predecessor node because due to overtaking node 6 moved out of node $k^{\prime}$ s transmission range. But node 4 which has lower speed than node 6 (see dashed line at node $7^{\prime}$ s arrival) falls in node $7^{\prime}$ s transmission range and the two nodes can communicate directly.

In sparse networks, the mobility of nodes is considered independent of each other. However, in dense networks, such an assumption is not valid ${ }^{[8]}$. Consider the following assumptions:

1) Nodes enter the hazard segment following a Poisson distribution with arrival rate $\lambda$ (vehicles/s).

2) Node mobility is random and independent.

We assume that node speeds are uniformly distributed in $\left[v_{\min }, v_{\max }\right]$, where $v_{\min }$ and $v_{\max }$ represent the minimum and the maximum speeds on the highway, respectively.

\subsection{The proposed model}

Focusing on the instant a vehicle enters the hazard segment, we evaluate the following metrics in our analytical model:

1) Joining probability, which is defined as the probability that a vehicle receives the message and joins the informed platoon.

2) Expected informed platoon size, which is defined as the expected number of consecutive informed vehicles.

3) Expected message propagation delay in the platoon, which is defined as the expected time to propagate the message to all the vehicles in the informed platoon using the store-and-forward scheme.

The first one is important, because a higher joining probability means a higher chance for a vehicle to get informed of the condition in the hazard region. The second one is important, because it shows how many informed vehicles enter the hazard segment. Studying the last metric gives us an insight into the parameters affecting the propagation 


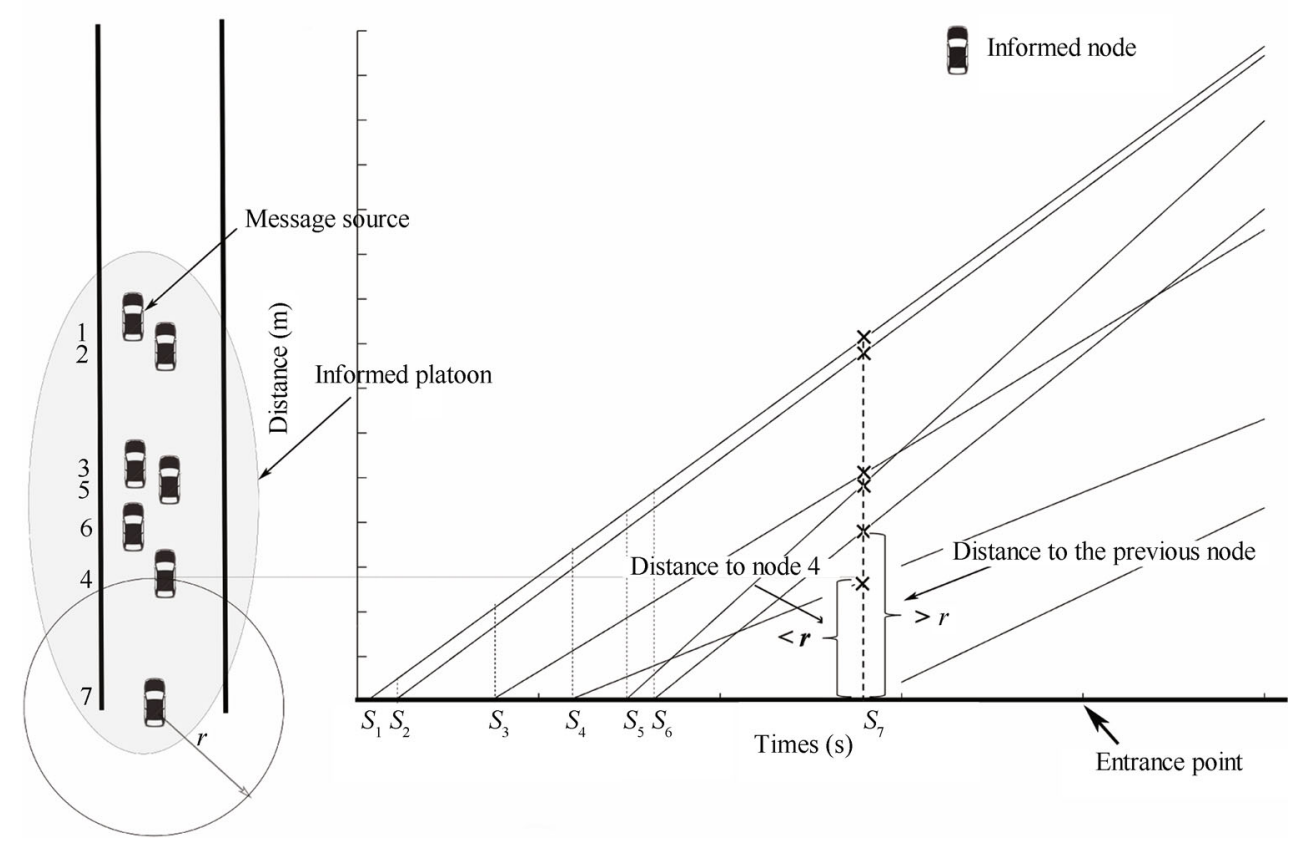

Fig. 1 Vehicle trajectories in non-stationary traffic

delay in store-and-forward inter-vehicle communication, which is discussed in Subsection 3.2.3.

\subsubsection{Joining probability}

In this subsection, we obtain the joining probability of node $k$ as follows ${ }^{[21]}$ :

Let $T_{i}, i=1,2, \cdots, N(t)$ be a random variable $(\mathrm{RV})$ denoting inter-arrival time between nodes $i$ and $i-1$. According to the Poissonly distributed arrival of nodes assumed in Subsection 3.1, we know that $T_{i}$ s are independent and identically distributed exponential RVs with $\mathrm{E}\left[T_{i}\right]=\frac{1}{\lambda}$ given by

$$
f_{T_{i}}\left(t_{i}\right)=\lambda \mathrm{e}^{-\lambda t_{i}}, \quad i=1,2, \cdots, N(t), t_{i}>0 .
$$

Assuming that $N(t)$ nodes enter the hazard segment in $[0, t]$, first we find the joint probability density function (PDF) of $k$ arrival times, where $k \leq N(t)$, meaning that we release the condition $N(t)=k$ on the joint PDF of arrival times which is assumed in Theorem 5.2 of [22]. According to [22], it is known that

$$
k \leq N(t) \Leftrightarrow S_{k} \leq t
$$

The following lemma holds:

Lemma 1. The joint PDF of arrival times $S_{1}, S_{2}, \cdots, S_{k}, k \leq N(t)$ can be expressed as

$$
\begin{array}{r}
f_{S_{1}, S_{2}, \cdots, S_{k}}\left(s_{1}, s_{2}, \cdots, s_{k}\right)=\lambda^{k} \mathrm{e}^{-\lambda s_{k}} \\
0<s_{1}<\cdots<s_{k-1}<s_{k} \leq t .
\end{array}
$$

Proof. We know that the inter-arrival times until node $k^{\prime}$ s arrival (i.e., $T_{i}, i=1, \cdots, k$ ) are independent and identically distributed with a PDF given by (1). Thus, the joint
PDF of these RVs can be expressed as

$$
\begin{aligned}
& f_{T_{1}, T_{2}, \cdots, T_{k}}\left(t_{1}, t_{2}, \cdots, t_{k}\right)=f_{T_{1}}\left(t_{1}\right) f_{T_{2}}\left(t_{2}\right) \cdots f_{T_{k}}\left(t_{k}\right)= \\
& \quad \lambda \mathrm{e}^{-\lambda t_{1}} \lambda \mathrm{e}^{-\lambda t_{2}} \cdots \lambda \mathrm{e}^{-\lambda t_{k}} \\
& 0<t_{i} \leq t
\end{aligned}
$$

The arrival times until node $k^{\prime}$ s arrival (i.e., $S_{i}, i=$ $1, \cdots, k)$ can be defined as

$$
\begin{aligned}
S_{1}= & T_{1} \\
S_{2}= & T_{1}+T_{2} \\
& \cdots \\
S_{k}= & T_{1}+T_{2}+\cdots+T_{k} .
\end{aligned}
$$

The joint PDF of arrival times is a multivariate linear transformation of the joint PDF of inter-arrival times having an absolute value of the Jacobian of the transformation 1. It means

$$
f_{S_{1}, S_{2}, \cdots, S_{k}}\left(s_{1}, s_{2}, \cdots, s_{k}\right)=f_{T_{1}, T_{2}, \cdots, T_{k}}\left(t_{1}, t_{2}, \cdots, t_{k}\right)
$$

where

$$
\begin{aligned}
t_{1}= & s_{1} \\
t_{2}= & s_{2}-s_{1} \\
& \cdots \\
t_{k}= & s_{k}-s_{k-1} .
\end{aligned}
$$

Therefore,

$$
\begin{gathered}
f_{S_{1}, S_{2}, \cdots, S_{k}}\left(s_{1}, s_{2}, \cdots, s_{k}\right)= \\
\lambda \mathrm{e}^{-\lambda s_{1}} \mathrm{e}^{-\lambda\left(s_{2}-s_{1}\right)} \times \cdots \\
\mathrm{e}^{-\lambda\left(s_{k}-s_{k-1}\right)}=\lambda^{k} \mathrm{e}^{-\lambda s_{k}} \\
0<s_{1}<s_{2}<\cdots<s_{k} \leq t .
\end{gathered}
$$


Let $X_{k-i}$ be an RV representing the waiting time from the $i$-th entrance until the $k$-th entrance, i.e.,

$$
X_{k-i}=S_{k}-S_{i}
$$

In order to have a linear transformation from a $k$ dimensional space to a $k$-dimensional space, we introduce an additional new RV as

$$
X_{k}=S_{k} .
$$

The corresponding $X_{i}, i=1, \cdots, k$ for the example shown in Fig. 1 is illustrated in Fig. 2. As can be seen, the $X_{i}$ s are arranged in an ascending order as $S_{i}$ s are.

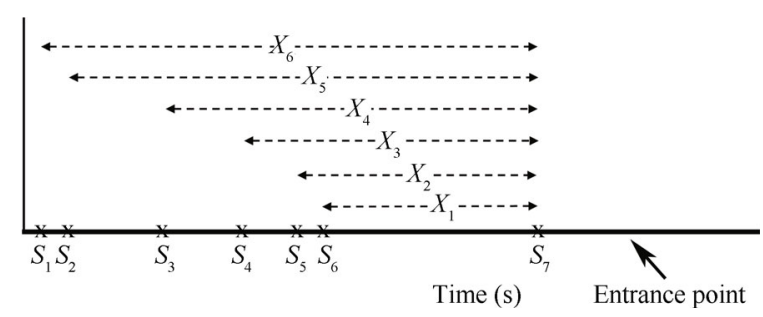

Fig. $2 X_{i}, i=1,2, \cdots, k$ for the example shown in Fig. 1

Since the absolute value of the Jacobian of the transformation is 1 , the joint PDF of waiting times (i.e., $X_{k-i}$ ) is the same as (3) and can be defined as

$$
\begin{gathered}
f_{X_{1}, X_{2}, \cdots, X_{k}}\left(x_{1}, x_{2}, \cdots, x_{k}\right)=\lambda^{k} \mathrm{e}^{-\lambda x_{k}}, \\
0<x_{1}<\cdots<x_{k-1}<x_{k} \leq t .
\end{gathered}
$$

The marginal PDF of $X_{k-i}$ is obtained as

$$
\begin{aligned}
& f_{X_{k-i}}\left(x_{k-i}\right)= \\
& \quad \int \cdots \int_{\mathcal{D}} \lambda^{k} \mathrm{e}^{-\lambda x_{k}} \mathrm{~d} x_{1} \cdots \mathrm{d} x_{k-i-1} \mathrm{~d} x_{k-i+1} \cdots \mathrm{d} x_{k}
\end{aligned}
$$

where $\mathcal{D}=\left\{\left(x_{1}, x_{2}, \cdots, x_{k}\right): 0<x_{1}<x_{2}<\cdots<x_{k} \leq t\right\}$.

Define

$$
Z_{i, k}=X_{i-k} V_{i}
$$

where $Z_{i, k}$ is an RV representing the distance between node $i$ and node $k$, and $V_{i}$ is an RV representing node $i^{\prime}$ s speed having a PDF given by

$$
f_{V_{i}}\left(v_{i}\right)=\frac{1}{v_{\max }-v_{\min }}, \quad v_{\min } \leq v \leq v_{\max }
$$

which is identical for all the nodes. Let $P\left(k_{c_{i}}\right)$ denote the contact probability of node $k$ with a predecessor node $i, i=1,2, \cdots, k-1$, i.e., the probability that node $k$ communicates with node $i$ when it enters the highway segment. Let $P\left(k_{c}\right)$ denote the total contact probability of node $k$, i.e., the probability that node $k$ finds at least one predecessor node in its transmission range when it enters the highway segment. Since $V_{i}$ and $X_{k-i}$ are independent RVs, the probability that node $i$ is in the transmission range of node $k$, i.e., $P\left(Z_{i, k} \leq r\right)$ by applying the conditional distribution formula ${ }^{[23]}$, is obtained as

$$
\begin{aligned}
& P\left(k_{c_{i}}\right)=P\left(Z_{i, k} \leq r\right)= \\
& P\left(X_{k-i} V_{i} \leq r\right)= \\
& \quad \int_{-\infty}^{+\infty} P\left(X_{k-i} V_{i} \leq r \mid V_{i}=v_{i}\right) f_{V_{i}}\left(v_{i}\right) \mathrm{d} v_{i}= \\
& \int_{v_{\min }}^{v_{\max }} P\left(X_{k-i} \leq \frac{r}{v_{i}}\right) \times \frac{1}{v_{\max }-v_{\min }} \mathrm{d} v_{i}, i<k .
\end{aligned}
$$

$P\left(X_{k-i} \leq \frac{r}{v_{i}}\right)$ can be obtained by using (12), and $P\left(k_{c}\right)$ is obtained as

$$
\begin{aligned}
& P\left(k_{c}\right)=P\left\{\cup_{i=1}^{k-1} A_{i, k}\right\}= \\
& P\left\{A_{1, k} \cup A_{2, k} \cup \cdots \cup A_{k-1, k}\right\}= \\
& \quad \sum_{i} P\left(A_{i, k}\right)-\sum_{i<j} P\left(A_{i, k} A_{j, k}\right)+ \\
& \quad \sum_{i<j<l} P\left(A_{i, k} A_{j, k} A_{l, k}\right)+\cdots+ \\
& (-1)^{k} P\left(A_{1, k} A_{2, k} \cdots A_{k-1, k}\right), \quad k=2,3, \cdots, N(t)
\end{aligned}
$$

where $A_{i, k}$ denotes event $Z_{i, k} \leq r, i=1, \cdots, k-1, P\left(A_{i, k}\right)$ can be obtained by (15), and $P\left(A_{i, k} \cdots A_{j, k} \cdots A_{m, k}\right), i<$ $\cdots<j<\cdots<m$ can be obtained by (17) at the next page. Since node speeds are independent, we have (18) at the next page, where $P\left\{X_{k-i} \leq \frac{r}{v_{i}}, \cdots, X_{k-j} \leq \frac{r}{v_{j}}, \cdots, X_{k-m} \leq \frac{r}{v_{m}}\right\}$ is obtained from (19) at the next page, where $\quad f_{X_{k-i}, X_{k-j}, \cdots, X_{k-m}}\left(x_{k-i}, \cdots, x_{k-j}, \cdots, x_{k-m}\right)$ is the marginal PDF of $n$-dimensional RV $\left(x_{k-i}, \cdots, x_{k-j}, \cdots, x_{k-m}\right)$, and $\mathcal{D}^{\prime \prime}$ is a subset of $\mathcal{D}^{\prime}$ shown by (20) at the next page.

Node $k$ becomes an informed node and joins the informed platoon if nodes $2,3, \cdots, k$ contact with at least one informed node as they enter the highway segment (i.e., $\left.j_{c}, j=2,3, \cdots, k\right)$. The informed node stores and carries the message for a while and hands it to the approaching vehicle as it appears on the highway segment. For example in Fig. 1, node 2 is an informed node since it has the message source in its transmission range. It stores and carries the message and forwards it when a new node appears on the highway segment. When node 3 enters the highway segment, it has 2 informed nodes in its transmission range. Therefore, it joins the informed platoon. It carries the message and forwards it when a new communication link appears. Finally, node 7 joins the informed platoon, since it has node 4 in its transmission range, which is an informed node. Thus, the probability that node $k$ receives the message and joins the informed platoon can be obtained as

$$
\begin{aligned}
& P\left(k_{\text {join }}\right)=P\left\{\cap_{j=2}^{k}\left(j_{c}\right)\right\}= \\
& P\left\{\cap_{j=2}^{k}\left(\cup_{i=1}^{j-1} A_{i, j}\right)\right\}= \\
& P\left\{A_{1,2} \cap\left(A_{1,3} \cup A_{2,3}\right) \cap \cdots\right\}
\end{aligned}
$$

where $P\left(k_{\text {join }}\right)$ denotes the probability that node $k$ joins the 


$$
\begin{aligned}
& P \overbrace{\left(A_{i, k} \cdots A_{j, k} \cdots A_{m, k}\right)}^{v_{\max }}=P\left(Z_{i, k} \leq r, \cdots, Z_{j, k} \leq r, \cdots, Z_{m, k} \leq r\right)= \\
& \quad \int_{v_{\min }}^{v_{\max }} P\left\{\int_{v_{\min }} X_{k-i} V_{i} \leq r, \cdots, X_{k-j} V_{j} \leq r, \cdots, X_{k-m} V_{m} \leq r \mid V_{i}=v_{i}, \cdots, V_{j}=v_{j}, \cdots, V_{m}=v_{m}\right\} \times \\
& \quad f\left(v_{i}, \cdots, v_{j}, \cdots, v_{m}\right) \mathrm{d} v_{i}, \cdots, \mathrm{d} v_{j}, \cdots, \mathrm{d} v_{m}, \quad i<\cdots<j<\cdots<m<k \\
& P\left(A_{i, k}, \cdots, A_{j, k}, \cdots, A_{m, k}\right)=\int_{v_{\min }}^{v_{\max }} \cdots \int_{v_{\min }}^{v_{\max }} P\left\{X_{k-i} \leq \frac{r}{v_{i}}, \cdots, X_{k-j} \leq \frac{r}{v_{j}}, \cdots, X_{k-m} \leq \frac{r}{v_{m}}\right\} \times \\
& \quad\left(\frac{1}{v_{\max }-v_{\min }}\right)^{n} \mathrm{~d} v_{i}, \cdots, \mathrm{d} v_{j}, \cdots, \mathrm{d} v_{m} \\
& P\left\{X_{k-i} \leq \frac{r}{v_{i}}, \cdots, X_{k-j} \leq \frac{r}{v_{j}}, \cdots, X_{k-m} \leq \frac{r}{v_{m}}\right\}= \\
& \quad \int \cdots \int_{\mathcal{D}^{\prime \prime}} f_{X_{k-i}, X_{k-j}, \cdots, X_{k-m}}\left(x_{k-i}, \cdots, x_{k-j}, \cdots, x_{k-m}\right) \mathrm{d} x_{k-i}, \cdots, \mathrm{d} x_{k-j}, \cdots, \mathrm{d} x_{k-m} \\
& \mathcal{D}^{\prime}=\left\{\left(x_{1}, x_{2}, \cdots, x_{k-1}\right): 0<x_{1}<x_{2}<\cdots<x_{k-1} ; x_{k-1} \leq \frac{r}{v_{1}} ; x_{i} \leq \min _{j=i, \cdots, k-1} \frac{r}{v_{k-j}}, i=1, \cdots, k-2\right\}
\end{aligned}
$$

informed platoon. The above equation can be obtained by using (16).

Approximated formulas. In this subsection, we propose simple approximated formulas for (16) and (21) which are difficult to obtain especially when the number of nodes increases. Simulation results show that contact probability of node $k$, i.e., $P\left(k_{c}\right)$ greatly depends on node $k-1$, slightly on node $k-2$ and very slightly on the other nodes. If we assume that only node $k-1$ has impact on $P\left(k_{c}\right)$, then (16) can be approximated by

$$
P\left(k_{c}\right) \approx P\left(A_{k-1, k}\right) .
$$

where $P\left(A_{k-1, k}\right)$ denotes event $Z_{k-1, k} \leq r$ (when the distance between two consecutive nodes $k-1$ and $k$ is less than or equal to the transmission range). It is concluded that vehicle contact can be assumed as an independent RV, thus

$$
P\left(k_{\text {join }}\right) \approx P\left\{A_{1,2} \cap A_{2,3} \cap A_{3,4} \cap \cdots\right\} \approx \prod_{j=2}^{k} P\left(A_{j-1, j}\right) .
$$

This approximation is useful for further analysis of the message propagation process especially in finding the expected message propagation delay in the platoon.

\subsubsection{Expected informed platoon size}

Using the approximated formulas obtained in the previous subsection, we find the expected informed platoon size, i.e., the expected number of consecutive informed nodes that enter the highway segment. As discussed earlier, if node $k$ contacts with an informed node with probability of $P\left(k_{c}\right)$ it becomes informed as well. Otherwise, with $1-P\left(k_{c}\right)$ it is an uninformed node and the informed node chain breaks. The number of informed nodes can be expressed as an RV having a geometric distribution with $P_{n c}=1-P\left(k_{c}\right)$. Based on the approximation in Sub- section 3.2.1, $P\left(k_{c}\right) \approx P\left(A_{k-1, k}\right)$. Since node contact is an independent and identically distributed RV, the probability mass function (PMF) of the number of informed nodes can be written as

$$
P\left(N_{I}=n_{i}\right)=P_{n c}\left(1-P_{n c}\right)^{n_{i}-1}
$$

where $N_{I}$ is an RV denoting the number of informed nodes, and the expected informed platoon size is

$$
\mathrm{E}\left[N_{I}\right]=\frac{1}{P_{n c}} .
$$

\subsubsection{Expected message propagation delay in the platoon}

In this subsection, we find the average time to propagate the message to all the vehicles in the platoon. Clearly, the average time to deliver the message to the informed platoon tail gives us the expected message propagation delay in the informed platoon. The message propagation delay in a mobile network using store-and-forward consists of two parts: 1) communication delay, when communication path is available between two nodes; 2) carry delay, when the message is carried by a vehicle until a new communication path appears. In VANETs, the carry delay is several orders-of-magnitude larger than the communication delay. Therefore, we ignore the communication delay and consider the carry delay in order to find the expected message propagation delay in the platoon. The desired metric can be obtained as follows.

The PDF of inter-arrival time between two consecutive informed nodes can be obtained as

$f_{T_{\text {inf }}}\left(t_{\text {inf }}\right)=f_{T}\left(t \mid T \leq \frac{r}{V}\right)= \begin{cases}\frac{f_{T}(t)}{F_{T}\left(\frac{r}{V}\right)}, & \text { if } 0 \leq t \leq \frac{r}{v_{\min }} \\ 0, & \text { otherwise }\end{cases}$

where $T_{\mathrm{inf}}$ is an RV denoting the inter-arrival time between two consecutive informed nodes. Since the inter-arrival 
times as well as the node speeds are independent, in the above equation, we dropped the subscripts. $F_{T}\left(\frac{r}{V}\right)$ can be obtained using (15), and the average inter-arrival time between two consecutive informed nodes can be obtained as

$$
\mathrm{E}\left[T_{\mathrm{inf}}\right]=\int_{0}^{\frac{r}{v_{\min }}} t_{\text {inf }} f\left(t_{\mathrm{inf}}\right) \mathrm{d} t_{\text {inf }} .
$$

The message propagation delay in the platoon, $T_{p d}$, is

$$
T_{p d}=\sum_{i=0}^{N_{I}-1} T_{\inf _{i}} .
$$

Therefore, the expected message propagation delay in the platoon is obtained as

$$
\mathrm{E}\left[T_{p d}\right]=\mathrm{E}\left[N_{I}-1\right] \mathrm{E}\left[T_{\mathrm{inf}}\right]=\left(\mathrm{E}\left[N_{I}\right]-1\right) \mathrm{E}\left[T_{\mathrm{inf}}\right] .
$$

\subsection{Discussion}

A typical scenario of our model is presented in Fig. 3. Since the distance between node 8 (newly entered) and node 7 (informed node) is greater than the transmission range, the message cannot be relayed to node 8 and it stays uninformed and does not join the informed platoon. Therefore, node 7 is the last informed node that enters the highway hazard segment (i.e., informed platoon tail). Also, due to the traffic dynamics, it is possible that node 8 joins the informed platoon later. This phenomenon is not discussed here and we leave it as future works. Since we do not consider communication delay in our model, the expected sum of the arrival times conditioned on $T \leq \frac{r}{V}$, from the message source's arrival to node $7^{\prime}$ s arrival gives us the expected message propagation delay in the platoon. This means that the message propagation delay with store-and-forward policy is tightly related to traffic parameters such as traffic flow rate and node speeds on the highway, also it is related to the transmission range of communicating vehicles and the MPR as well.

In bidirectional highway scenarios, in a case that the connectivity is not available in the original direction, vehicles traveling in the opposite direction can help to restore the connectivity. In this case, when the message source observes a hazard on the road, it may transfer the message to a vehicle driving in the opposite direction. This vehicle (relay vehicle) carries the message and forwards it to a vehicle in the original direction when the communication link appears. In safety applications, this piece of information is of no use for the relay vehicles. Therefore, the message must return to the original direction before vehicles in the original direction reach the defined distance threshold. In sparse networks which we study in this paper, due to low network density, vehicles in the opposite direction cannot help message propagation in the original direction remarkably and they are more useful in networks between sparse and dense ${ }^{[4,19]}$.

\section{Numerical and simulation results}

In this section, the theoretical results are validated through simulation. Using Matlab, entrance to the highway segment is simulated for a sufficiently large time interval (i.e., 1000s). The highway is assumed to be unidirectional and having multiple lanes, but there is no restriction on the number of lanes, i.e., nodes can overtake without changing their lanes or maneuvering. Due to the small width of the lanes, this assumption does not affect the connectivity and the error is negligible. Traffic flow assumed to be undisturbed (i.e., no traffic lights). It is assumed that the

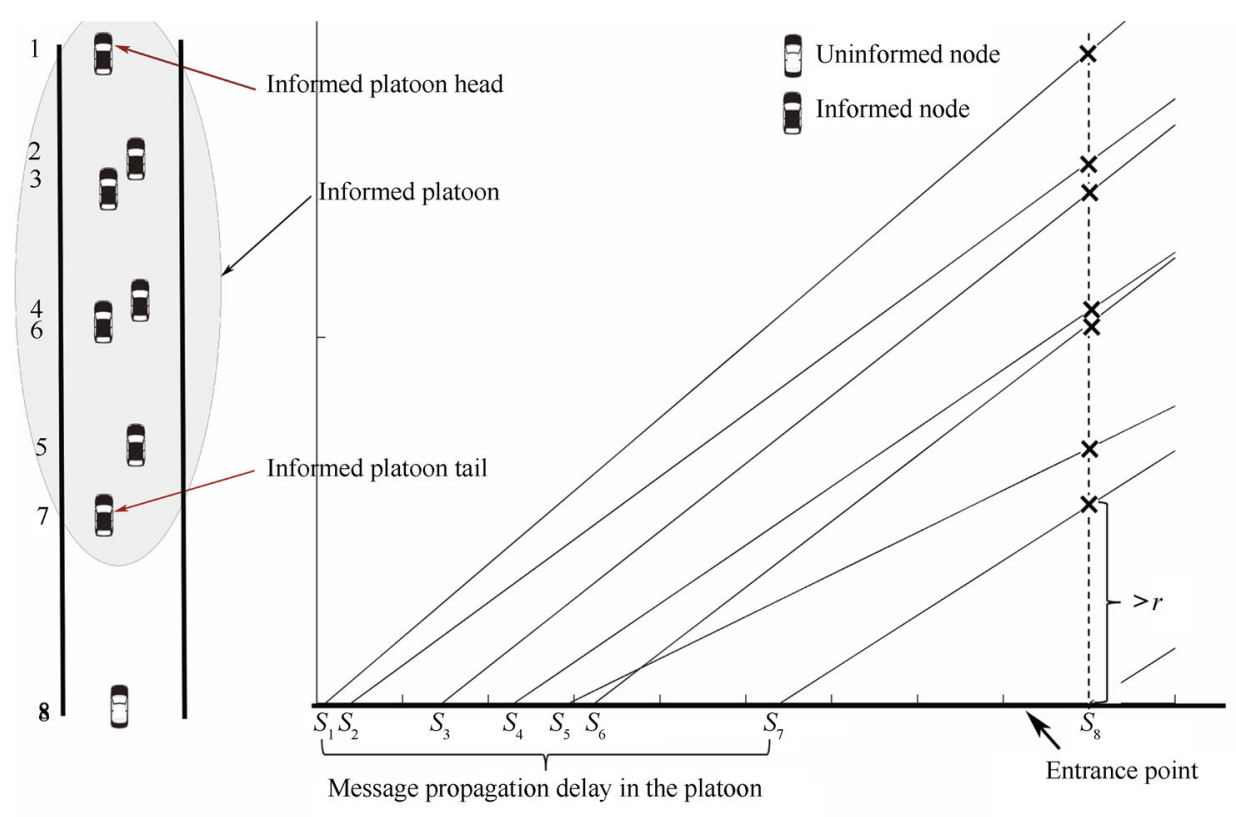

Fig. 3 Illustrative scenario of the proposed model 
highway segment is long enough compared to the transmission range, i.e., the message propagation is not spatially restricted. According to [7, 17], it is known that when the traffic volume is below $1000 \mathrm{veh} / \mathrm{h}$, the network formed by vehicles is sparse. Therefore, the traffic volume is considered to be 430 vehicles/h which is an empirical value measured in [17]. As a result, the traffic flow rate $\lambda$ is 0.12 vehicles/s. Node speeds are uniformly distributed in $\left[v_{\min }, v_{\max }\right]$ interval, where $v_{\min }=20 \mathrm{~m} / \mathrm{s}$ and $v_{\max }=40 \mathrm{~m} / \mathrm{s}$. Nodes do not change their chosen speeds over the simulation time. The simulation is repeated for a sufficiently large number of trials by allowing random number of nodes enter the highway segment in the simulation time in each trial, and statistics of the desired performance measure for node $k$ is collected. The desired probabilities are obtained from the ratio $n_{\text {desired }} / n_{\text {total }}$, where $n_{\text {desired }}$ is the number of trials in which the desired event has occurred and $n_{\text {total }}$ is the total number of trials.

In Fig. 4 , the contact probability of node $k$ with a predecessor node $i(i=1,2, \cdots, k-1)$ as a function of transmission range, which is given in (15), is evaluated against the simulation results. In fact, it shows the chance of the newly entered node being able to communicate with the predecessor nodes. In Fig. $4, k$ is 7 , the 1st predecessor node is vehicle 6 , the 2 nd predecessor node is vehicle 5 , etc. Obviously, increasing the transmission range may increase the chance of having more nodes in the transmission range. For example, for a transmission range of $300 \mathrm{~m}$, which is the current feasible transmission range ${ }^{[24]}$, the chance of having the third predecessor node in node $7^{\prime} \mathrm{s}$ transmission range is about $15 \%$, but for a transmission range of $1000 \mathrm{~m}$, which is the proposed transmission range of the dedicated shortrange communication (DSRC) standard ${ }^{[25,26]}$, it is about $75 \%$.

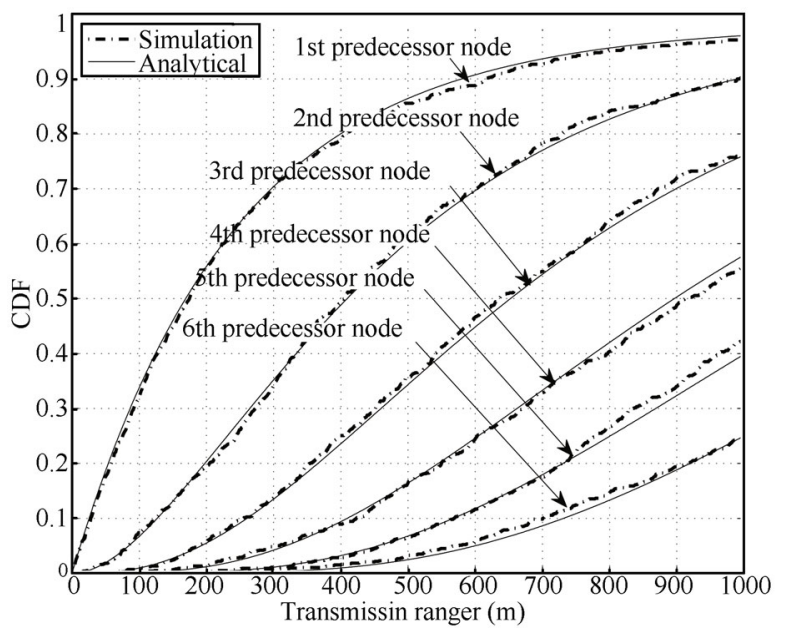

Fig. 4 Contact probability of node $k(k=7)$ with a predecessor node $i(i=1,2, \cdots, 6)$

Fig. 5 shows the total contact probability obtained in (16). As can be seen, node 3 (with two predecessor nodes, i.e., nodes 1 and 2 ) compared to node 2 (with only one predecessor node, i.e., node 1) benefits from a higher contact probability. In fact, it shows the effectiveness of non-stationary traffic in the message propagation process. Hence, as the node number increases, the total contact probability of the node increases as well. But for nodes after the third, the total contact probability increases very slightly, showing that the nodes except the two predecessor nodes have little impact on the total contact probability. This fact led us to the approximated formulas in Subsection 3.2.1. However, the node transmission range has a great impact on the total contact probability. As can be observed, for smaller transmission ranges (e.g., $100 \mathrm{~m}$ ), approximation results, obtained by (22), are in good agreement with the analytical and simulation results. In other word, for smaller transmission ranges, node $k$ contacts mostly with node $k-1$, while for larger transmission ranges, a disagreement between the approximation results with the analytical and simulation results is observed. It means that node $k$ may contact with other predecessor nodes when the transmission range is larger. Thus, an increase in the analytical and simulation results compared to the approximation results is observed (see the slope of the line from node 2 to node 3 for different transmission ranges in Fig. 5).

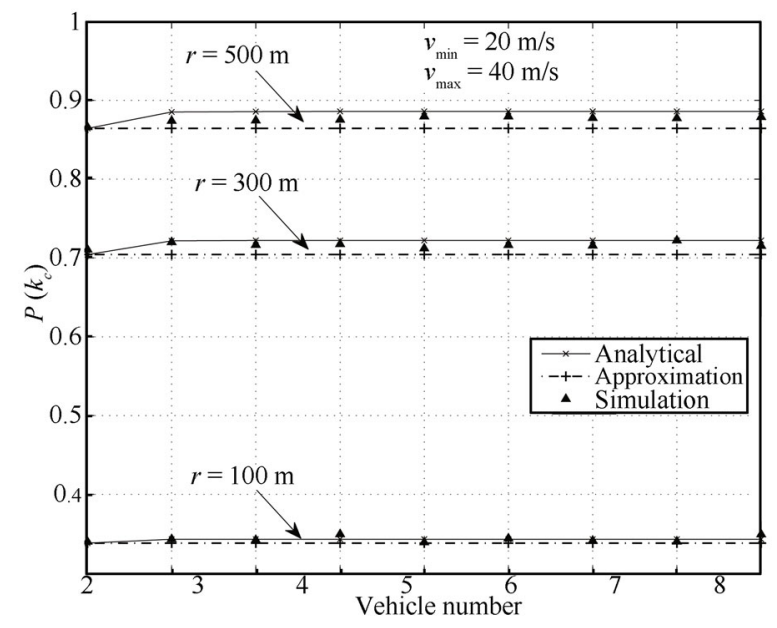

Fig. 5 Total contact probability of node $k, k=2, \cdots, 10$

Fig. 6 shows the probability that node $k$ joins the informed platoon $P\left(k_{\text {join }}\right)$ when it enters the highway segment for three different transmission ranges. As can be seen, for a given transmission range, as $k$ increases, the joining probability decreases. This is due to the fact that for node $k$, to join the informed platoon, all the predecessor nodes and node $k$ must contact with an informed node. Therefore, as the number of node $k$ increases, this probability decreases. On the other hand, increasing the transmission range increases this probability. As can be observed, for smaller transmission ranges, there is a good agreement between the analytical and simulation results with approximation results. But for larger transmission ranges as discussed in Fig. 5, a node may contact with other predecessor nodes and the independency assumption does not hold any more. Hence, a disagreement between the analytical and simula- 
tion results with approximation results is evident.

Fig. 7 shows the impact of road speed limits on $P\left(k_{\text {join }}\right)$. As may be observed, by reducing the road maximum speed limit to $30 \mathrm{~m} / \mathrm{s}$, the probability that node $k$ joins the informed platoon increases compared to the maximum speed limit of $40 \mathrm{~m} / \mathrm{s}$. As can be seen, there is a good agreement between the approximation results with simulation and analytical results when $v_{\max }$ is reduced to $30 \mathrm{~m} / \mathrm{s}$.

Fig. 8 shows the average informed platoon size for different transmission ranges and different arrival rates (i.e., traffic flow) in a sparse network (from 430 vehicles/h to 720 vehicles/h). As may be observed, for small transmission ranges (e.g., $100 \mathrm{~m}$ ), small platoons are formed. Even increasing the traffic flow does not increase the platoon size very much. But, for larger transmission ranges (e.g., $300 \mathrm{~m}$, $500 \mathrm{~m}$ ) the contact probability is high, therefore, more vehicles tend to join the informed platoon and larger platoons are formed. In this situation, even a small increase in the traffic flow may increase the platoon size. This increase in the platoon size is more remarkable for much larger transmission ranges (i.e., for $r=500 \mathrm{~m}$ compared to $r=300 \mathrm{~m}$ in Fig. 5). As can be seen, for larger transmission ranges, there is a disagreement between the analytical results and the simulation results (e.g., $500 \mathrm{~m}$ ). This inaccuracy stems from the fact that for larger transmission ranges, the impact of other nodes on a node's contact increases and the approximation in Subsection 3.2.1 does not remain accurate. As a result, the obtained analytical results are not in good agreement with the simulation results.

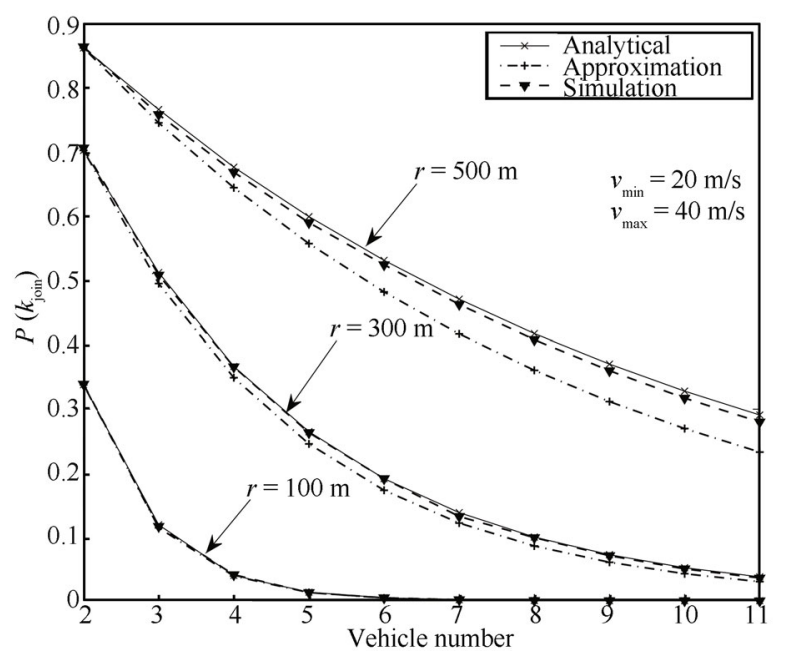

Fig. 6 Probability that node $k(k=2,3, \cdots, 10)$ joins the informed platoon $P\left(k_{\text {join }}\right)$ for three different transmission ranges $(r=100 \mathrm{~m}, 300 \mathrm{~m}$, and $500 \mathrm{~m})$ with a maximum speed limit of $40 \mathrm{~m} / \mathrm{s}$

Fig. 9 shows the expected message propagation delay in the platoon as a function of arrival rate for different transmission ranges. For small transmission ranges (e.g., $100 \mathrm{~m}$ ) since small platoons are formed, the expected message propagation delay in the platoon is low. But, as discussed in Fig. 8, for larger transmission ranges (e.g., $300 \mathrm{~m}, 500 \mathrm{~m}$ ) larger platoons are the formed. As a result, message propagation in the platoon takes more time and even a small increase in the traffic flow rate may increase the expected propagation delay in the platoon, which is more noticeable for the larger transmission range (e.g., $500 \mathrm{~m}$ ). Also, we see a disagreement between the analytical results and the simulation results as the transmission range increases for the same reason discussed in Fig. 8.

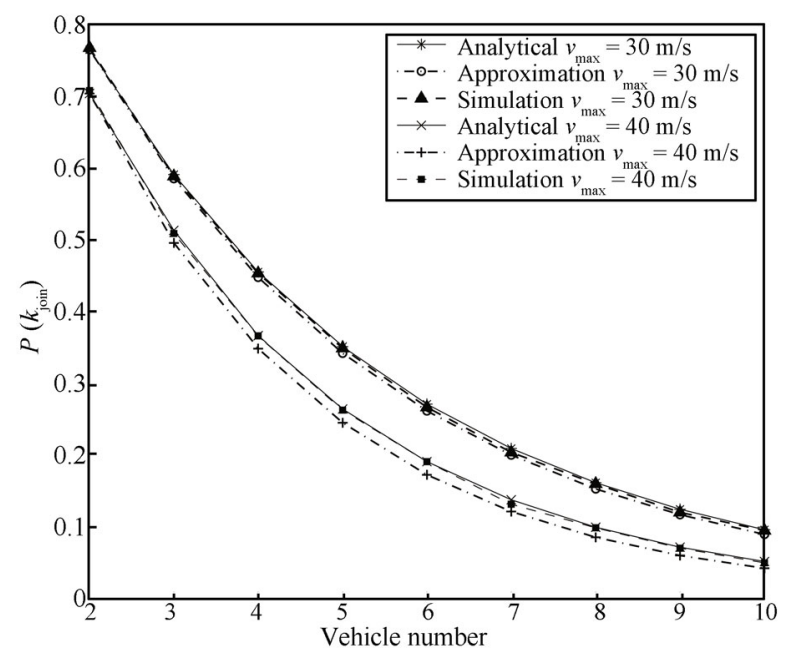

Fig. 7 Probability that node $k(k=2,3, \cdots, 10)$ joins the informed platoon, $P\left(k_{\text {join }}\right)$, with transmission range $r=300 \mathrm{~m}$ for two different road speed limits (i.e., $v_{\min }=20 \mathrm{~m} / \mathrm{s}, v_{\max }=$ $30 \mathrm{~m} / \mathrm{s}$ and $v_{\min }=20 \mathrm{~m} / \mathrm{s}, v_{\max }=40 \mathrm{~m} / \mathrm{s}$ )

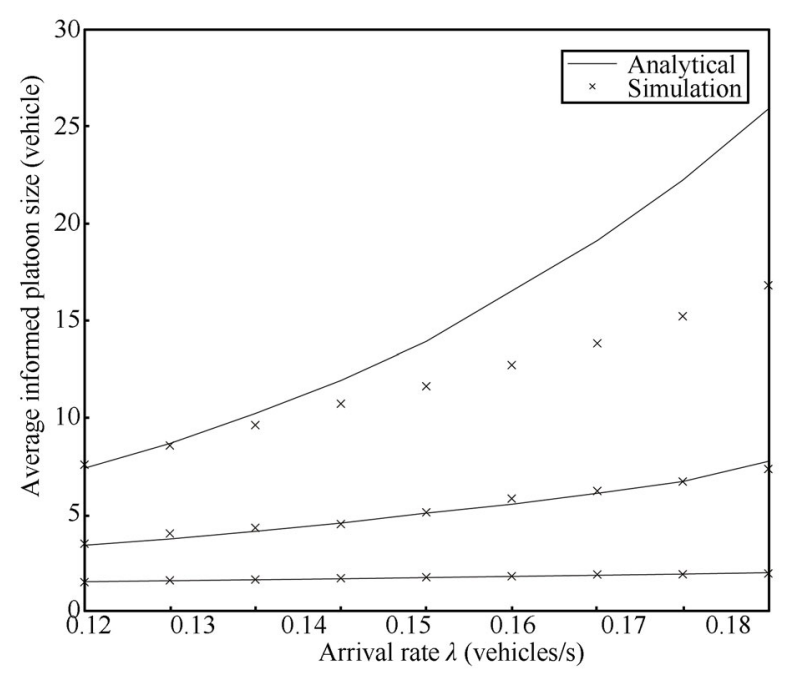

Fig. 8 Average informed platoon size

\section{Conclusions and future works}

In this paper, we studied the propagation of road hazard information to the vehicles which enter the hazard segment using store-and-forward in a sparse VANET. We obtained the probability that a given vehicle receives the message from the message source and joins the platoon of informed vehicles. In particular, the fact that traffic dynamics increases the reception probability of messages was proven 


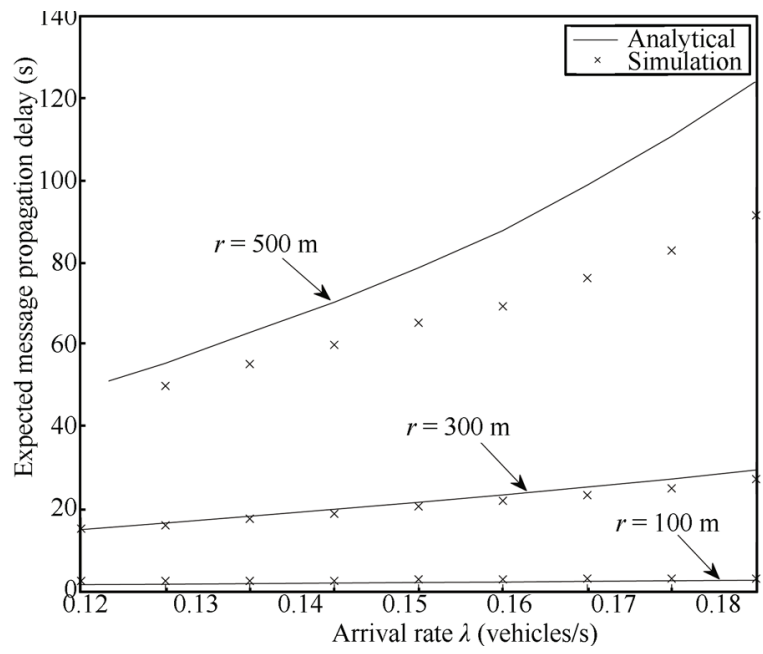

Fig. 9 Expected message propagation delay in the platoon

here. We found that the total contact probability of a node largely depends on node $k-1$, slightly on node $k-2$ and very slightly on the other nodes. Thus, we proposed an approximated formula for the total contact probability of a node. This formula was further used for finding two important performance measures, i.e., expected informed platoon size and expected message propagation delay in the platoon. We found that the expected message propagation delay is related to traffic parameters such as traffic flow rate, node speeds on the highway and the transmission range of equipped vehicles. Results showed that for smaller transmission ranges, smaller platoons are formed and the expected platoon size and the expected message propagation delay in the platoon increase very slightly as the traffic flow increases. But for larger transmission ranges, larger platoons are formed and the expected size and the expected delay increase noticeably even with a small increase in the traffic flow.

The future plan is: 1) to extend the model and study store-and-forward message propagation in VANETs considering MAC related problems and 2) to study store-andforward message propagation in bidirectional and more realistic traffic scenarios.

\section{References}

[1] A. Casteigts, A. Nayak, I. Stojmenovic. Communication protocols for vehicular ad hoc networks. Wireless Communications and Mobile Computing, vol.11, no. 5, pp. 567-582, 2011.

[2] L. H. Immers, S. Logghe. Traffic Flow Theory, Faculty of Engineering, Department of Civil Engineering, Section Traffic and Infrastructure, Katholieke Universiteit Leuven, Heverlee, Belgium, 2002.

[3] H. Wu, R. M. Fujimoto, G. F. Riley, M. Hunter. Spatial propagation of information in vehicular networks. IEEE Transactions on Vehicular Technology, vol. 58, no. 1, pp. 420-431, 2009.
[4] W. L. Jin, W. Recker. An analytical model of multihop connectivity of intervehicle communication systems. IEEE Transactions on Wireless Communications, vol.9, no. 1, pp. 106-112, 2010.

[5] M. Desai, D. Manjunath. On the connectivity in finite ad hoc networks. IEEE Communications Letters, vol. 6, no. 10, pp. 437-439, 2002.

[6] A. Ghasemi, S. Nader-Esfahani. Exact probability of connectivity in one-dimensional ad hoc wireless networks. IEEE Communications Letters, vol. 10, no. 4, pp. 251-253, 2006.

[7] S. Yousefi, E. Altman, R. El-Azouzi, M. Fathy. Analytical model for connectivity in vehicular ad hoc networks. IEEE Transactions on Vehicular Technology, vol. 57, no. 6, pp. 3341-3356, 2008.

[8] J. X. Wu. Connectivity of mobile linear networks with dynamic node population and delay constraint. IEEE Journal on Selected Areas in Communications, vol. 27, no. 7, pp. 1218-1225, 2009.

[9] M. Khabazian, M. K. Ali. A performance modeling of connectivity in vehicular ad hoc networks. IEEE Transactions on Vehicular Technology, vol. 57, no. 4, pp. 2440-2450, 2008.

[10] G. H. Mohimani, F. Ashtiani, A. Javanmard, M. Hamdi. Mobility modeling, spatial traffic distribution, and probability of connectivity for sparse and dense vehicular ad hoc networks. IEEE Transactions on Vehicular Technology, vol. 58, no. 4, pp. 1998-2007, 2009.

[11] R. Groenevelt, P. Nain, G. Koole. The message delay in mobile ad hoc networks. Performance Evaluation, vol. 62, no. $1-4$, pp. 210-228, 2005.

[12] W. Zhao, M. Ammar, E. Zegura. A message ferrying approach for data delivery in sparse mobile ad hoc networks. In Proceedings of ACM International Symposium on Mobile Ad Hoc Networking and Computing, ACM, New York, USA, pp. 187-198, 2004.

[13] Q. Li, D. Rus. Sending messages to mobile users in disconnected ad hoc wireless networks. In Proceedings of $A C M$ International Conference on Mobile Computing and Networking, ACM, New York, USA, pp. 44-55, 2000.

[14] D. Nain, N. Petigara, H. Balakrishnan. Integrated routing and storage for messaging applications in mobile ad hoc networks. Mobile Networks and Applications, vol.9, no. 6, pp. 595-604, 2004.

[15] A. Agarwal, D. Starobinski, T. D. Little. Exploiting downstream mobility to achieve fast upstream message propagation in vehicular ad hoc networks. In Proceedings of IEEE Mobile Networking for Vehicular Environments, Anchorage, Alaska, pp. 13-18, 2007.

[16] S. Panichpapiboon, W. Pattara-atikom. Connectivity requirements for self-organizing traffic information systems. IEEE Transactions on Vehicular Technology, vol. 57, no. 6, pp. 3333-3340, 2008. 
[17] N. Wisitpongphan, F. Bai, P. Mudalige, V. Sadekar, O. Tonguz. Routing in sparse vehicular ad hoc wireless networks. IEEE Journal on Selected Areas in Communications, vol. 25, no. 8, pp. 1538-1556, 2007.

[18] A. Kesting, M. Treiber, D. Helbing. Connectivity statistics of store-and-forward inter-vehicle communication. IEEE Transactions on Intelligent Transportation Systems, vol. 11, no. 1, pp. 172-181, 2010.

[19] A. Agarwal, D. Starobinski, T. D. C. Little. Phase transition of message propagation speed in delay tolerant vehicular networks. IEEE Transactions on Intelligent Transportation Systems, vol. 13, no. 1, pp. 249-263, 2012.

[20] R. P. Roess, E. S. Prassas, W. R. McShane. Traffic Engineering, 3rd ed., Englewood Cliffs, NJ, USA: Prentice-Hall, 2004.

[21] S. Keykhaie, M. Dehghan, S. Yousefi. Probability of multihop message dissemination in sparse linear vehicular ad hoc networks. In Proceedings of IEEE International Conference on Vehicular Electronics and Safety, IEEE, Istanbul, Turkey, pp. 105-110, 2012.

[22] S. M. Ross. Introduction to Probability Models, 9th ed., New York, USA: Academic Press, 2007.

[23] A. M. Mood, F. A. Graybill, D. C. Boes. Introduction to the Theory of Statistics, New York, USA: McGraw-Hill, 1974.

[24] U. S. Department of Transportation. Vehicle Safety Communications Project, Final Report, Public Document, Crash Avoidance Metrics Partnership, 2006.

[25] Federal Communications Commission, FCC 03-324. FCC Report and Order, 2004.

[26] IEEE. IEEE Standard 802. 11p Draft Amendment, Wireless LAN Medium Access Control (MAC) and Physical Layer (PHY) Specifications: Wireless Access in Vehicular Environments (WAVE), 2005.

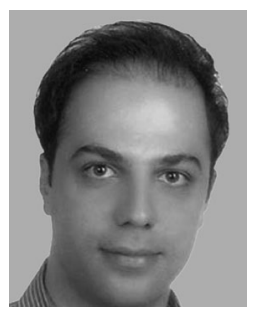

Sepehr Keykhaie received the B.Sc. degree in computer engineering from Islamic Azad University of Najafabad (IAUN), Iran in 2005, and the M. Sc. degree in computer networking from Islamic Azad University of Qazvin (QIAU), Iran in 2011. He joined the Department of Computer and IT Engineering of Chehelsotoun Institute of Higher Education, Iran in 2012. He is now the vice head of the institute.

His research interests include performance modeling, VANETs, (vehicular) delay tolerant networks, (vehicular) social networks, and intelligent transportation systems.

E-mail: sepehr.keykhaie@gmail.com (Corresponding author) ORCID iD: 0000-0003-4873-2553

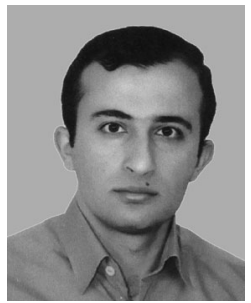

Saleh Yousefi received the B.Sc. and $\mathrm{M}$. Sc. degrees in computer engineering (in the hardware engineering field) and Ph. D. degree in computer engineering (in the networking field) from Iran University of Science and Technology, Iran in 1999, 2002 and 2008 , respectively. He is now an assistant professor at Department of Computer, Faculty of Engineering, Urmia University, Iran.

His research interests include the performance evaluation of computer networks, telecommunications in intelligent transportation systems, and (vehicular) ad hoc networks.

E-mail: s.yousefi@urmia.ac.ir

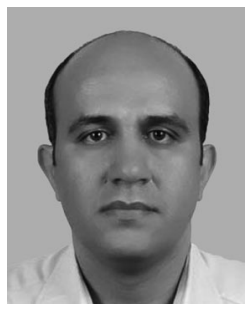

Mehdi Dehghan received the B.Sc. degree in computer engineering from Iran University of Science and Technology (IUST), Iran in 1992, and the M. Sc. and $\mathrm{Ph}$. D. degrees from Amirkabir University of Technology (AUT), Iran in 1995, and 2001 , respectively. He is now an associate professor at Department of Computer Engineering and IT, Amirkabir University of

Technology, Iran.

His research interests include high speed networks, network management, mobile ad hoc networks, and fault tolerant computing.

E-mail: dehghan@ce.aut.ac.ir 